\title{
Assuring Access to Abortion
}

\author{
Rachel Rebouché, JD, LLM, Temple University, Beasley School of Law
}

SUMMARY. Over the spring of 2020, numerous states announced measures suspending abortions in response to COVID-19. Banning abortion during the pandemic is counterproductive. Impeding access to abortion will not help preserve healthcare resources. Moreover, prohibiting access to abortion care exacerbates the strain on the healthcare system. People who lack access to abortions will travel to neighboring states, induce their own abortions, or carry pregnancies to term, which will require prenatal care and assistance in childbirth. Perhaps more importantly, the people hit hardest by suspending abortion care are those for whom the pandemic already has had devastating effects. Lifting restrictions on medication abortion and expanding telehealth abortion services will conserve healthcare resources and improve public health. Recognizing the advantages of telemedicine, some states, as well as the federal government, have relaxed restrictions on remote diagnosis and treatment. However, many of those same states have carved out exceptions for abortion in their telemedicine policies. In addition, people seeking medication abortions still face unnecessary restrictions on access, none of which are applied to comparable office-based procedures. Policymakers can eliminate barriers to safe abortion services now and in the future. "No-touch" terminations, in which all medical supervision happens over the telephone or online, can better accomplish the goals that the present abortion suspensions cannot. Telehealth for medical abortion can ease the burdens on pregnant people, healthcare workers, and health systems in light of the unprecedented challenges presented by COVID-19.

\section{Introduction}

Twelve states suspended abortion care, for differing lengths of time, in response to COVID-19 (Sobel et al., 2020). State officials argued that the policies classifying abortion as a nonessential surgery reduced patient-physician contact as well as preserved medical supplies, hospital space, healthcare capacity. All but two courts were unpersuaded by these arguments and issued injunctions of the orders after holding that the bans violated patients' constitutional right to an abortion, ignored medical evidence on the short-term and long-term consequences of delayed abortion care, and exacerbated the public health emergency by ultimately increasing pregnant people's use of healthcare systems.

This Chapter summarizes 12 states' classification of abortion as non-essential health care during the onset of the pandemic. It then examines the present restrictions on medication abortion that undermine efforts to curb the spread of COVID-19. Given the challenges presented by COVID-19, state and federal policy should permit teleabortion to the extent it is feasible and suspend medically unnecessary requirements, such as in-person consultations and pre-abortion ultrasounds, that increase clinicpatient contact. Enabling remote access to abortion would ease the already-heavy burdens that fall disproportionately on low income and people of color, whose lack of access to abortion has deep and longstanding health effects.

\section{State Abortion Care Suspensions}

In March and April of 2020, 12 states issued executive orders and public health directives that either implicitly or explicitly suspended abortion services during the COVID-19 emergency. In all but one state (Arkansas), these policies were enjoined by courts, lifted after settlements with state officials, or expired when executive orders expired.

\section{COVID-19 Orders Blocked through Litigation}

Four states implemented executive orders, issued by the governor or the state's public health department, that limited access to or resulted in a complete ban on abortion services. The following states' policies were enjoined in litigation in which courts held that suspension of non-essential services did not apply to abortion and the bans contravened women's constitutional rights to abortion before viability.

In Alabama, the state's public health officer issued an order on March 27, 2020, which postponed surgical procedures not necessary to treat an "emergency medical condition" or "avoid serious harm from an underlying condition." Abortion providers won a temporary restraining order on March 30, 2020, in federal district court, which the Court of Appeals for the Eleventh Circuit affirmed. At the end of April 2020, an amended order permitted surgical procedures that included abortion care. 
In Ohio, on March 17, 2020, the director of the state health department prohibited all nonessential surgeries and procedures that utilized personal protective equipment (PPE), including abortion services. The state's attorney general sought to enforce the order against abortion providers through cease and desist letters. Providers sued for a preliminary injunction, and the federal district court ruled that physicians may determine, on a case-bycase basis, whether an abortion procedure was "necessary because of the timing vis-à-vis pre-viability; to protect the patient's health or life; and due to medical reasons...." The Court of Appeals for the Sixth Circuit affirmed, holding that the order erected an "undue burden" on the constitutional right to abortion. By May 1, 2020, a new order reinstated all non-essential surgeries and procedures, including abortion.

On March 15, Oklahoma's governor issued an executive order postponing all elective surgeries and minor medical procedures. A state press release interpreted the order to apply to all abortions unless the procedures were necessary to prevent serious health risks or in response a medical emergency. Providers won a temporary restraining order that created exceptions for medication abortion and for patients nearing the gestational legal limit; the Court of Appeals for the Tenth Circuit affirmed. All abortion services resumed on April 24, 2020 when some elective surgeries resumed.

The governor of Tennessee issued an executive order that prohibited procedures, including abortion services, that were not necessary to address a medical emergency or to preserve the health and safety of a patient as determined by a licensed medical provider. Providers filed for a preliminary injunction, which the district court granted on April 17, 2020, on constitutional grounds. The Court of Appeals for the Sixth Circuit affirmed.

Texas' legal path was particularly twisting, and the litigation over the state's abortion suspension illustrates the arguments for and against the banning abortion as a pandemic-prevention measure. The governor issued an executive order on March 22, 2020 mandating all licensed health care professionals and facilities postpone surgeries and procedures not immediately medically necessary. The state's attorney general applied the order to abortion care unless there was a threat to the life of the pregnant person, and the Texas Medical Board issued an emergency rule giving the attorney general's interpretation effect. At the end of March 2020, a federal district court granted a temporary restraining order, which the Court of Appeals for the Fifth Circuit reversed. The Fifth Circuit held that Texas's abortion ban was a reasonable way to conserve medical supplies and hospital capacity. Moreover, the Fifth Circuit determined that medication abortion, which only entails taking two pills, reduces supplies of PPE because of the ultrasound and in-person consultation Texas law requires of all abortion patients. The district court granted a second temporary restraining order on April 9, 2020, permitting medication abortion and abortion for patients nearing gestational legal limit. Ultimately after another round of opinions, the Fifth Circuit reversed the second order, which resulted in the abortion suspension, with one exception, resuming. The Fifth Circuit again held that medication abortion consumed PPE, this time because providers (as other healthcare professionals) wear protective masks and gloves when seeing patients during the pandemic. The revived suspension was short-lived, however; two days later, a new executive order took effect, and the governor's office issued a statement that abortion was excluded from the order's terms.

\section{COVID-19 Orders that Expired or Were Replaced}

Alaska's Health Mandate, issued by the governor and the Department of Public Health on April 7, 2020, declared surgical abortions "non-urgent" and ordered them postponed unless the pregnancy endangered the woman's "life or physical health." The order remained in effect until it expired May 4, 2020. Kentucky's state legislature then passed a bill to limit access to abortion services. The governor of Kentucky vetoed the bill after the legislative session.

Mississippi's governor issued an order on April 10, 2020 that delayed all non-essential elective surgeries and medical procedures, including abortion services. The ban remained in effect without challenge until an updated order issued on May 11, 2020, which allowed abortion services to resume. lowa's governor issued an executive order on March 26, 2020 prohibiting all nonessential and elective surgeries and procedures that utilize PPE, including abortions. In lieu of a lawsuit, abortion providers and the government reached a settlement allowing abortion procedures to continue. Similarly, the Louisiana Department of Health's March 21, 2020 order postponed medical and surgical abortions for 30 days, except those (1) "to treat an emergency medical condition" or (2) "to avoid further harms from underlying condition or disease," leaving that determination to the provider's "best medical judgment." After the attorney general sent state representatives to observe abortion clinics' compliance with the order, abortion providers filed a legal challenge that was withdrawn after the parties reached a settlement that permitted abortion services to resume. In West Virginia, on March 31, 2020, the governor issued an executive order prohibiting all elective medical procedures that were not medically necessary to preserve the patient's life or long-term health, which the attorney general interpreted to include abortion services. The state's only abortion provider filed a federal lawsuit, but a new executive order on April 30, 2020 lifted the abortion suspension.

Finally, Arkansas was the state with the longest-lasting COVID-19 order limiting abortion. From April 10, 2020 until June 1, 2020, the Arkansas Department of Health banned "surgical" abortions except if necessary to protect the life or health of the patient. Providers filed for a temporary restraining order on April 13, 2020, which the district court granted. The Court of Appeals for the Eighth Circuit, however, reversed and also denied providers' request for an exception for patients approaching the gestational legal limit. The Eighth Circuit held that suspending abortion was a reasonable means to conserve hospital space and PPE, following the Fifth Circuit's reasoning described above. The state issued a modified order on April 27, 2020 allowing access to abortion services if patients had "at least one negative COVID-19 NAAT test within 48 hours prior to the beginning of the procedure." The Department of Health order was modified on May 18,2020, extending the testing timeframe to 72 hours, and the testing requirement was lifted on June 12, 2020 when the order expired. 
During the weeks of fluctuating legal status across these 12 states patients had their appointments cancelled with a moment's notice and were turned away from clinics (Alexandria, 2020). Clinics that reopened had long waiting lists for appointments. The resulting hardships of state abortion suspensions, affirm that, for patients with delayed or denied care, abortion is an essential service.

\section{Strain on the Healthcare System and Deepened Disparities}

Suspending abortion does not conserve scarce medical resources and does not impede COVID-19's spread. Banning abortion has the net effect of a greater consumption of health resources because people will travel out of state for abortion care, self-induce terminations, or will be forced to carry pregnancies to term.

First, many people who lack access to abortion will travel to other jurisdictions to end their pregnancies, consuming the same medical resources but requiring providers in neighboring states-without the assistance of additional staff or capacity-to manage an influx of new patients (Bearak et al., 2020). Already overextended providers saw an influx of new patients. As a result, wait times and crowding increased at clinics in states neighboring those with abortion suspensions. Increased delay comes with the cost of more expensive procedures later in pregnancy or timing out of a legal abortion altogether. And to emphasize what may be obvious, during the pandemic, people who travel long distances for abortion care cannot limit social contact and take risks that could be avoided but for their state's animus for abortion rights.

People who did not or could not travel, likely terminated pregnancies by ordering online (or procured elsewhere) one or both of the pills taken in a medication abortion and taking them without physician supervision. Self-managed abortion can be effective and safe. However, it can also increase costs for the healthcare system if patients lack accurate information and adverse health consequences occur.

Finally, and perhaps most significantly, unplanned parenthood results in the consumption of healthcare resources. Continuing a pregnancy requires prenatal care that includes multiple interactions, each necessitating PPE, with healthcare professionals-far more PPE, hospital space, and healthcare professionals' time than any type of abortion. Furthermore, childbirth has steep costs and health risks, particularly for low income people and people of color. The United States has the worst maternal mortality rate in comparison to countries similarly situated; Black women are four times as likely to die in childbirth than white women (Greene Foster, 2020).

In the same vein, abortion suspensions have fallen disproportionately on people who have shouldered the hardships imposed by COVID-19people who are unemployed or essential workers, and those who do not have access to healthcare or face other logistical challenges. These populations cannot afford the additional costs imposed by abortion suspensions, and they are people for whom COVID-19 has deepened unequal access to health resources.
Rather than suspend abortion, expanding access to medication abortion, particularly through telemedicine, could help slow COVID19 's spread. However, as the next section makes plain, longstanding regulation that contradicts medical evidence and clinical practice makes delivering medication abortion unnecessarily difficult.

\section{The Battle over Remote Abortion Care}

Abortion has been more closely regulated than comparable (and riskier) outpatient procedures well before COVID-19. Public health research makes clear that abortion-targeted laws, unlike rules for outpatient procedures with similar, or even higher risk, apply "regardless of the level of sedation or anesthesia used[ ] or the nature of the office intervention" (Jones et al., 2018).

Legislative efforts, in response to and before the onset of the pandemic, target medication abortion to undermine abortion rights rather than ensure patient safety or to conserve effectively health system and provider capacity. Contrary to the conclusions of the Fifth and Eighth Circuits, described above, medication abortions typically require no gown, mask, eyewear, shoe covers, or gloves; in other words, no PPE is used. Like the vast majority of terminations, medication abortion is not administered in a hospital or physician's office but in standalone clinics devoted to reproductive health services. Because the risks and complications associated with medication abortion are very low, rarely will a hospital bed be taken because of medication abortion (Upadhyay et al., 2019). Medication abortion could require no contact with healthcare providers, except that law requires it.

\section{Legal Restrictions on Teleabortion}

Despite the ease with which medication abortion can be administered, and its proven effectiveness, several states and the federal government obstruct efforts to provide remote solutions for its delivery. Medication abortions make up almost $40 \%$ of the nation's total abortions (Jones et al., 2019). In a medication abortion, which occurs during the first 10 weeks of pregnancy (or 11 weeks for off-label but accepted use), patients ingest two pills: the first drug, mifepristone, is followed by a second drug, misoprostol, taken 24-48 hours later. Federal rules prohibit dispensing the drugs through the mail or at a pharmacy. The U.S. Food and Drug Administration (FDA) restricts mifepristone under a drug safety program (a Risk Evaluation and Mitigation Strategy or REMS), which mandates, among other things, collection of the drug at a clinic, physician's office, medical center, or hospital.

Also, several states' laws impose additional restrictions beyond the REMS protocol. Eighteen states mandate that the prescribing physician be physically present when the patient collects and takes the medication (LawAtlas State Abortion Laws, 2019). The number of states mandating the physical presence of a physician during medication abortion will increase if pending state bills pass. In addition, 33 states prohibit non-physicians from administering medication abortion despite evidence that advanced practice clinicians can safely and effectively counsel patients. These laws layer on top of additional legal requirements, such as pre-termination ultrasounds and counseling. Mandatory ultrasound requirements specifically thwart teleabortion by necessitating clinic-patient contact. 
Finally, nine states ban telehealth through legislation that exempts abortion from any permitted telemedicine. On the federal level, a bill before Congress, the Teleabortion Prevention Act of 2020, excludes abortion services from telehealth measures by requiring that physicians be present during terminations. At the same time, the federal government has expanded telehealth for non-abortion medical services, recognizing the importance of health care solutions that limit contact between professionals and patients (Ross, 2020). The coronavirus relief legislation issued guidelines for Medicaid and Medicare coverage of telehealth and included grants to develop telehealth practices for federally qualified health centers, rural health clinics, and hospices. Likewise, 27 states have extended telemedicine, through new legislation or executive orders, as a response to the pandemic (see Schmit et al., Chapter 16). For example, the same week that the Texas Medical Board issued an emergency rule to apply suspend abortion as non-essential care, the same board relaxed restrictions on medical consultation, treatment, and diagnosis over the Internet and telephone.

\section{Support for Teleabortion}

Research demonstrates that medication abortion, like many other healthcare procedures, can be safely and effectively administered online or over the telephone. Teleabortion could permit "no-touch" terminations, which have demonstrated effectiveness and low risk to patients suitable for remote supervision (Raymond et al., 2019). Patients who are not at risk for medical complications, are less than eight weeks pregnant, and have regular menstrual cycles may not need in-person visits or pre-termination ultrasounds. A study launched by Gynuity Health Projects (with permission from the FDA) monitored healthcare professionals providing medication abortion care by videoconference and mail. Results of the study illustrate that "direct-to-patient telemedicine abortion service was safe, effective, efficient and satisfactory" (Raymond et al., 2019). A literature review summarizes that "there is overwhelming evidence that the safety and effectiveness of medication abortion is the same whether it is provided via telemedicine or through in-person provision, as shown by a seven-year cohort study with tens of thousands of patients, systematic reviews, and an evaluation of a telemedicine abortion service across five states" (Center for Reproductive Rights \& Columbia Mailman School of Public Health, 2020).

Some states, embracing this evidence, have recognized abortion as essential health care that must remain available during the national emergency. Three states explicitly protected access to abortion in executive orders, and an increasing number of health centers have relied on teleabortion, where permitted, so that eligible patients can pick up medication and self-administer while being in remote contact with their physician (Baker, 2020). As an early response to the pandemic, 21 state attorneys general wrote a letter urging the government to lift or to stop enforcing the FDA's protocol for mifepristone (Becerra et al., 2020).

Moreover, the call for teleabortion presently is before the federal judiciary. On July 13, 2020, the US District Court of the District of Maryland issued a nationwide injunction of the REMS mifepristone protocol for the duration of COVID-19 national emergency. The court noted that the REMS restriction contradicts substantial evidence of the drug's safety. The protocol also unreasonably singles out mifepristone without any corresponding health benefit. Of the 20,000 drugs regulated by the FDA, mifepristone is the only one that patients must retrieve at a medical center but may self-administer without supervision. In fact, the FDA permits mailing the same compound, when not prescribed for abortion or miscarriage, to patients' homes in higher doses and larger quantities. The effect of the REMS classification is that medication abortion cannot be mailed, excluding the possibility of telehealth for abortion. The FDA's enforcement of the in-person requirement for mifepristone stands in stark contrast to the numerous ways the FDA (as well as other federal agencies) have encouraged telemedicine as a response to the pandemic.

The decision also details the cumulative effects of abortion restrictions based on expert testimony and public health researchthat the "combination of such barriers can establish a substantial obstacle." The court cited evidence of how the in-person requirement exacerbates the burdens already felt by those who work essential jobs or are unemployed, have lost health insurance, live in multi-generational homes, and lack transportation. The opinion highlighted that low-income patients and people of color suffer disproportionately; they are more likely to become ill, to have inadequate resources to respond to illness, and will have worse health outcomes as a result deep health inequalities. Mirroring the logistical challenges most abortion patients face, the pandemic makes arranging for childcare, transport, or time off work especially difficult.

The district court's decision has been appealed to the Fourth Circuit, and it may come before the Supreme Court, depending on how long the national emergency lasts. 


\section{Recommendations for Action}

\section{Federal government:}

- The FDA should stop enforcing the outdated REMS protocol so that:

o Physicians no longer have to certify in a written form submitted to the drug sponsor that they have certain required qualifications;

o Mifepristone can be dispensed outside of a hospital, clinic, or medical office, by or under the supervision of a certified healthcare provider.

- The FDA should issue guidance confirming the results of studies demonstrating medication abortion's safety and efficacy, allowing mifepristone to be ordered through mail-order prescription services and at retail pharmacies.

- Congress should enact legislation that, counter to the Teleabortion Prevention Act 2020, advances teleabortion by recognizing that medical abortion can be a health service appropriately included in plans for telemedicine's expansion.

- Congress should pass a supplemental appropriations act for the Coronavirus Aid, Relief, and Economic Security Act (CARES Act) that does not exclude funding for teleabortion care.

\section{State governments:}

- Repeal cumbersome abortion regulations, such as waiting periods and ultrasound requirements, so that patients can avoid unnecessary visits to clinics and decrease the risk of COVID-19 exposure.

- Repeal penalties for self-managed abortion including criminal penalties for extralegal abortion.

- Repeal restrictions on telemedicine as applied to abortion, such as in-person and physician-only administration of medication abortion.

- Include medication abortion among the healthcare services subject to state efforts to expand telemedicine or to relax restrictions on telemedicine.

- Lift restrictions on telehealth modes (include telephone, audio-only communications), locations (permit use at home), delivery (allow any health care provider operating across jurisdictions) in revised state orders and legislation. 


\section{About the Author}

Rachel Rebouché, JD, LLM, is the Associate Dean for Research and James E. Beasley Professor of Law at Temple University, Beasley School of Law.

\section{References}

Alexandria, P. Abortion Is Available Again in Texas. But You'll Have to Get in Line. Rewire News. Retrieved July 28, 2020, from https://rewire.news/article/2020/05/06/abortion-isavailable-again-in-texas-but-youll-have-to-get-in-line/

Baker, C. N. (2020). Feminist Multi-Front Battle to End FDA's Abortion Pill Restriction. Ms. Magazine. Retrieved July 16, 2020, from https://msmagazine.com/2020/05/20/feministmulti-front-battle-to-end-fdas-abortion-pill-restriction/

Bearak, J., Jones, R. K., Nash, E., \& Donavan, M. K. (2020). Policy Analysis: COVID-19 Abortion Bans Would Greatly Increase Driving Distances for Those Seeking Care. Retrieved July 16, 2020 from the Guttmacher Institute, https://www.guttmacher.org/article/2020/04/ covid-19-abortion-bans-would-greatly-increase-driving-distances-those-seeking-care

Center for Reproductive Rights \& Columbia Mailman School of Public Health (2020). Expanding Telemedicine Can Ensure Abortion Access During COVID-19 Pandemic. Retrieved July 16, 2020, from https://reproductiverights.org/sites/default/files/documents/ Expanding\%20Telemedicine\%20Can\%20Ensure\%20Abortion\%20Access\%20During\%20 COVID-19\%20Pandemic.pdf

Greene Foster, D., Antonia Biggs, M., Ralph, L., Gerdts, C., Roberts, S., \& Maria Glymour, M (2018). Socioeconomic Outcomes of Women Who Receive and Women Who Are Denied Wanted Abortions in the United States. American Journal of Public Health, 108(3), 407-413.

Jones, B. S., Daniel, S., \& Cloud, L. K. (2018). State Law Approaches to Facility Regulation of Abortion and Other Office Interventions. American Journal of Public Health, 108(4), 486-492.

Jones, R. K., Witwer, E., \& Jerman, J. (2019). Abortion Incidence and Service Availability in the United States, 2017. Retrieved July 16, 2020 from the Guttmacher Institute, https:// www.guttmacher.org/report/abortion-incidence-service-availability-us-2017

LawAtlas (2019). State Abortion Laws, Medication Abortion Requirements. Retrieved July 28, 2020 from http://lawatlas.org/datasets/medication-abortion-requirements

Raymond, E., Chong, E., Winikoff, B., Platais, I., Mary, M., Lotarevich, T., Castillo, P. W., Kaneshiro, B., Tschann, M., Fontanilla, T., Baldwin, M., Schnyer, A., Coplon, L,Mathieu, N., Bednarek, P., Keady, M., \& Priegue, E. (2019). TelAbortion: evaluation of a direct to patient telemedicine abortion service in the United States. Contraception, 100(2019), 173-177.

Ross, J. (2020). Ohio Senate Passes Bill to Ban Use of Telemedicine for Abortions. Retrieved July 16, 2020 from Jurist, https://www.jurist.org/news/2020/03/ohio-senate-passes-billto-ban-use-of-telemedicine-for-abortions/

Sobel, L., Frederiksen, B., \& Salganicoff, A. (2020). State Action to Limit Abortion Access During the COVID-19 Pandemic. Retrieved July 16, 2020 from the Kaiser Family Foundation, https://www.kff.org/womens-health-policy/issue-brief/state-action-to-limit-abortionaccess-during-the-covid-19-pandemic/

Upadhyay, U. D., Desai, S., Zlidar, V., Weitz, T. A., Grossman, D., Anderson, P., \& Taylor, D. (2015). Incidence of Emergency Department Visits and Complications After Abortion. Obstetrics \& Gynecology, 125(1), 175-183.

Upadhyay, U. D., \& Grossman, D. (2019). Telemedicine for Medication Abortion. Contraception, 100(2019), 351-353.

Teleabortion Prevention Act of 2020, S. 3252, 116th Congress (2020), retrieved July 16, 2020 from https://www.congress.gov/bill/116th-congress/senate-bill/3252/text 